\title{
AB/Tubingen Zebrafish
}

National Cancer Institute

\section{Source}

National Cancer Institute. AB/T ubingen Zebrafish. NCI Thesaurus. Code C79956.

A zebrafish line established from a group of 25 crosses between individual $A B$ and

Tubing en fish. The progeny of two crosses that lacked recessive embryonic phenotypes through 5 or 6 days of development were selected as founders of two distinct lethal free lines that were designated TAB-5 and TAB-14. 